\title{
International cross-sectional survey on the use of webinars in plastic surgery: a move towards a hybrid educational model
}

\author{
Siri Gowda ${ }^{1}$ D . Jonathan Simmons ${ }^{1} \cdot$ Vimal J. Gokani $^{1}$
}

Received: 29 March 2021 / Accepted: 25 June 2021 / Published online: 2 August 2021

(c) Crown 2021

\begin{abstract}
Background The novel coronavirus pandemic (COVID-19) has catalyzed the development of online educational resources. Webinars provide opportunities for convenient distance-learning and provision of expert lectures, while reducing the financial and practical costs of attending conferences. This cross-sectional survey aims to collect subjective opinion on the usefulness of webinars as an educational platform compared to face-to-face interactions, and whether surgeons and surgeons-in-training want webinars to remain a permanent platform for training and development in the future.

Methods A free-to-attend online series of international expert lectures on a range of plastic surgery topics was delivered. Attendees were invited to anonymously complete a survey on attitudes towards webinars as an educational platform over a 2-week period.

Results A total of 883 complete responses were collected. Overall webinar attendance increased following the pandemic, with $97.4 \%$ of respondents reporting they view webinars for at least $1 \mathrm{~h}$ per week following implementation of COVID-19 restrictions. 90.4\% respondents indicated that they intend to continue utilizing webinars even once COVID-19 restrictions are eased, and $77.8 \%$ stated they learn as much from online webinars as in-person lectures. However, irrespective of training grade, a significant proportion of respondents believe webinars should not replace face-to-face meetings.

Conclusions This study provides evidence that, while face-to-face meetings remain a valued component of education in Plastic Surgery, webinars are a useful adjunct to conventional methods, and may feature more prominently in the educational landscape of the future.

Level of evidence: Not gradable.
\end{abstract}

Keywords Plastic surgery · Education · Webinar · COVID-19

\section{Introduction}

Over the past few decades, the use of technology in healthcare has facilitated rapid communication between clinicians, remote patient consultation, and distance learning. [1,2] In line with a global increase in the ownership of smartphones, and engagement with apps and social media, medical education in plastic surgery has also been trending towards online methods of delivery. [3, 4]

The novel coronavirus pandemic (COVID-19) has catalyzed the development of online educational resources.

Siri Gowda

siri.gowda@nhs.net

1 Department of Plastic and Reconstructive Surgery, Imperial College Healthcare NHS Trust, Fulham Palace Rd,

London W6 8RF, UK
Social distancing measures designed to curb viral transmission have resulted in prohibition of physical group interaction, and thereby changed the landscape of courses and conferences for the foreseeable future. The surgical profession has had to rapidly adapt by turning to alternative platforms for collaboration, clinical care, and education. This paper looks at the latter, and more importantly, at whether the surge of online learning should be the "new normal."

Webinars are online lectures delivered in real-time via web-based teleconferencing programs. [5] They provide opportunities for convenient distance-learning and provision of expert lectures, while reducing the financial and practical costs of attending conferences. [6] Following the COVID-19 pandemic, webinars in plastic surgery have been utilized to deliver educational content for specialist exams in addition to providing resources for medical students and doctors, during the prohibition of physical meetings. [7, 8] Although 
innovation is often borne out of necessity, the dependency on web-based learning as opposed to meetings remains to be determined. This cross-sectional survey aims to collect subjective opinion on the usefulness of webinars as an educational platform compared to face-to-face interactions, and whether surgeons and surgeons-in-training want webinars to remain a permanent platform for training and development in the future.

\section{Material and methods}

The weekly Imperial Plastic Surgery Webinars are a freeto-attend online series of international expert lectures on a range of plastic surgery topics, which started on 22nd April, 2020. Webinars were delivered using Zoom ${ }^{\mathrm{TM}}$ (Zoom Video Communications Inc., San Jose, CA, USA) on an evening (GMT) during the week, and lasted $1 \mathrm{~h}$ each. There was no cost associated with the organization, the speakers, to attend the lectures, or to subsequently view the recorded lectures on ImperialPlasticSurgery.com. The Zoom account, website domain name, and hosting were funded by the Imperial Plastic Surgery Unit.

All participants who registered to attend webinars between 16th April 2020 and 7th December 2020 were contacted via email and requested to anonymously complete an online survey designed using Survey Monkey ${ }^{\mathrm{TM}}$ (SurveyMonkey.com, Palo Alto, CA, USA) (Table 1). The survey tool was developed by the two authors (SG and VG). The survey tool comprised multiple choice questions, Likert scale responses, and free-text answers where applicable. Survey completion offered early access registration to the next 4 webinars, and the details of the website. The registration links were, however, openly published 1 to 2 weeks prior to the lectures, and nobody was denied access to the educational event. Access to survey completion was available between 10th December 2020 and 23rd December 2020, with 5 reminder emails within this period. Statistical analysis was performed using GraphPad Prism v9 (GraphPad Software, San Diego, CA, USA). Quantitative and qualitative methods were used to report on the survey data. No ethical approval was required for this study.

\section{Results}

A total of 883 complete responses to the survey were received; overall response rate was $31.4 \%$. Places of residence of respondents are shown in Fig. 1, and survey results are displayed in Tables 2, 3, 4, 5, 6, 7, 8, 9, 10, 11, 12, and 13.

Table 1 Webinar use survey, an optional 14th area for additional free-text comments was also provided

Q1. What is your current level of training?

Medical student/(Intern/Foundation Year/SHO/Core trainee)/(Resident/Specialty registrar)/(Attending/Consultant)

Q2. Which country do you currently practice in?

Select from drop down list of all countries

Q3. Prior to COVID-19 restrictions, how many hours did you spend attending webinars per week?

$0 / 1-2 / 3-5 / 6-10 />10$

Q4. Since COVID-19 restrictions, how many hours do you spend attending webinars per week?

$0 / 1-2 / 3-5 / 6-10 />10$

Q5. Do you mostly attend webinars during the working day or out-of-hours?

During working day/Out-of-hours/Both/Neither

Q6. Why do you attend webinars (tick all that apply)?

General knowledge or interest/Exam revision/Prominent speakers/CPD/Other (specify in additional comments box)

Q7. To what extent do you agree with the following statement: "I learn as much from webinars as I do from face-to-face lectures" Strongly agree/Agree/Neutral/Disagree/Strongly disagree

Q8. To what extent do you agree with the following statement: "I prefer live lectures to webinars"

Strongly agree/Agree/Neutral/Disagree/Strongly disagree

Q9. To what extent do you agree with the following statement: "I reflect on/discuss the webinars I attend with colleagues after I watch them" Strongly agree/Agree/Neutral/Disagree/Strongly disagree

Q10. To what extent do you agree with the following statement: "Webinars should replace face-to-face/departmental teaching" Strongly agree/Agree/Neutral/Disagree/Strongly disagree

Q11. To what extent do you agree with the following statement: "Webinars should replace face-to-face national and international meetings" Strongly agree/Agree/Neutral/Disagree/Strongly disagree

Q12. To what extent do you agree with the following statement: "I will continue to attend webinars once COVID-19 restrictions are lifted" Strongly agree/Agree/Neutral/Disagree/Strongly disagree

Q13. To what extent do you agree with the following statement: "I would pay to attend webinars given by high-quality speakers" Strongly agree/Agree/Neutral/Disagree/Strongly disagree 
Fig. 1 Infographic displaying place of residence of survey respondents weighted by prevalence, with the highest proportion of responses (30.43\%) from the UK

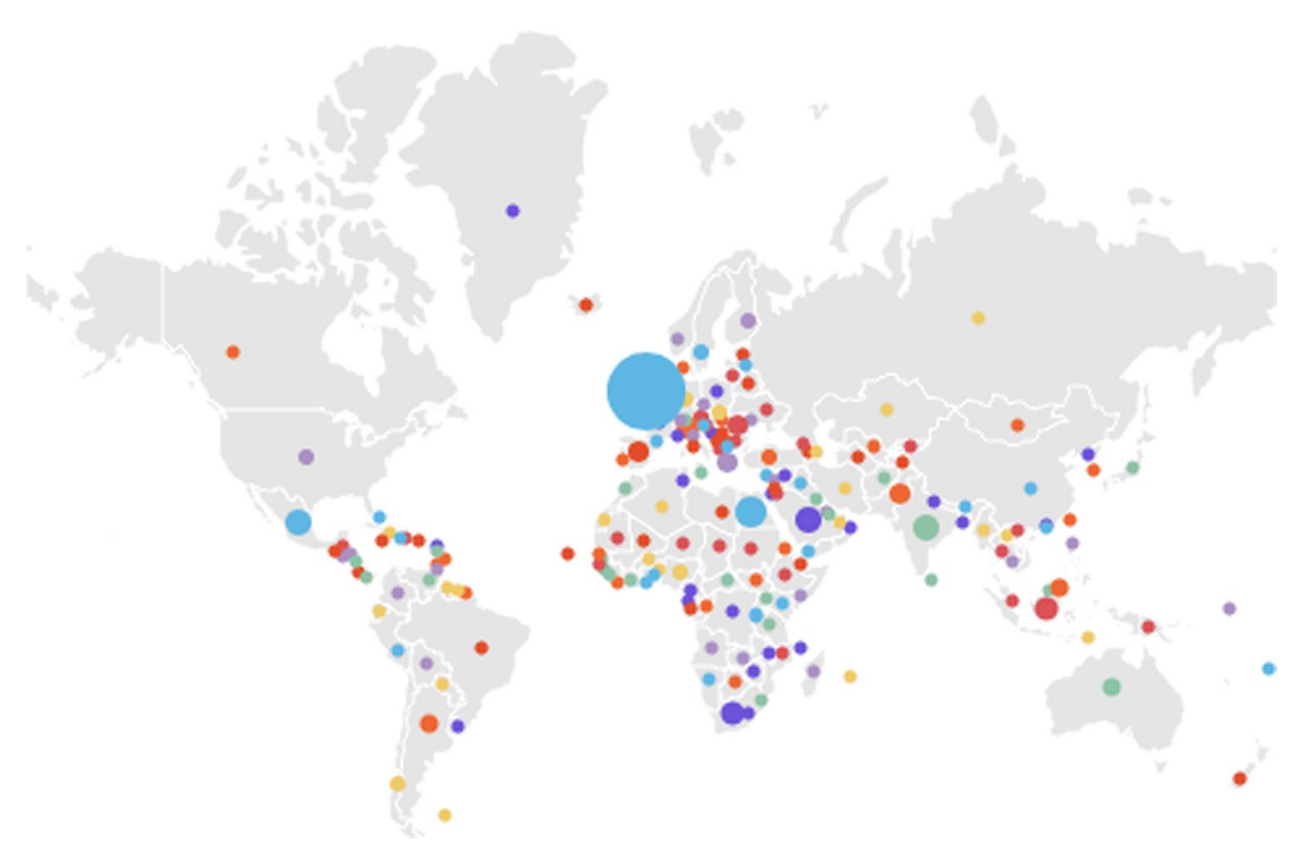

Table 2 What is your current level of training?

\begin{tabular}{llc}
\hline & $N$ responses & $\%$ responses \\
\hline Medical student & 18 & $2.04 \%$ \\
Intern/Foundation trainee/SHO/ & 56 & $6.34 \%$ \\
$\quad$ Core trainee & & \\
Resident/Specialty Registrar & 450 & $50.96 \%$ \\
Attending/Consultant & 359 & $40.66 \%$ \\
\hline
\end{tabular}

Table 3 Prior to COVID-19 restrictions, how many hours did you spend attending webinars per week?

\begin{tabular}{llc}
\hline & $N$ responses & $\%$ responses \\
\hline 0 & 536 & $60.70 \%$ \\
$1-2$ & 243 & $27.52 \%$ \\
$3-5$ & 76 & $8.61 \%$ \\
$6-10$ & 13 & $1.47 \%$ \\
Over 10 & 15 & $1.70 \%$ \\
\hline
\end{tabular}

Table 4 Since COVID-19 restrictions, how many hours do you spend attending webinars per week?

\begin{tabular}{llc}
\hline & $N$ responses & $\%$ responses \\
\hline 0 & 23 & $2.60 \%$ \\
$1-2$ & 366 & $41.45 \%$ \\
$3-5$ & 342 & $38.73 \%$ \\
$6-10$ & 113 & $12.80 \%$ \\
Over 10 & 39 & $4.42 \%$ \\
\hline
\end{tabular}

Table 5 Do you mostly attend webinars during the working day or out-of-hours?

\begin{tabular}{llc}
\hline & $N$ responses & $\%$ responses \\
\hline During working day & 32 & $3.62 \%$ \\
Out-of-hours & 601 & $68.06 \%$ \\
Both & 244 & $27.63 \%$ \\
Neither & 6 & $0.68 \%$ \\
\hline
\end{tabular}

Table 6 Why do you attend webinars (tick all that apply)?

\begin{tabular}{lll}
\hline & $N$ responses & $\%$ responses \\
\hline General knowledge/interest & 784 & $88.79 \%$ \\
Exam revision & 280 & $31.71 \%$ \\
Prominent speakers & 563 & $63.76 \%$ \\
CPD & 208 & $23.56 \%$ \\
Other (please specify) & 54 & $6.12 \%$ \\
\hline
\end{tabular}

Table 7 To what extent do you agree with the following statement: "I learn as much from webinars as I do from face-to-face lectures"

\begin{tabular}{llc}
\hline & $N$ responses & $\%$ responses \\
\hline Strongly agree & 248 & $28.09 \%$ \\
Agree & 439 & $49.72 \%$ \\
Neither agree nor disagree & 129 & $14.61 \%$ \\
Disagree & 62 & $7.02 \%$ \\
Strongly disagree & 5 & $0.57 \%$ \\
\hline
\end{tabular}


Table 8 To what extent do you agree with the following statement: "I prefer live lectures to webinars"

\begin{tabular}{llc}
\hline & $N$ responses & $\%$ responses \\
\hline Strongly agree & 105 & $11.89 \%$ \\
Agree & 253 & $28.65 \%$ \\
Neither agree nor disagree & 349 & $39.52 \%$ \\
Disagree & 158 & $17.89 \%$ \\
Strongly disagree & 18 & $2.04 \%$ \\
\hline
\end{tabular}

Table 9 To what extent do you agree with the following statement: "I reflect on/discuss the webinars I attend with colleagues after I watch them"

\begin{tabular}{llc}
\hline & $N$ responses & $\%$ responses \\
\hline Strongly agree & 123 & $13.93 \%$ \\
Agree & 479 & $54.25 \%$ \\
Neither agree nor disagree & 189 & $21.40 \%$ \\
Disagree & 77 & $8.72 \%$ \\
Strongly disagree & 15 & $1.70 \%$ \\
\hline
\end{tabular}

Table 10 To what extent do you agree with the following statement: "Webinars should replace face-to-face/departmental teaching"

\begin{tabular}{llr}
\hline & $N$ responses & $\%$ responses \\
\hline Strongly agree & 64 & $7.25 \%$ \\
Agree & 163 & $18.46 \%$ \\
Neither agree nor disagree & 294 & $33.30 \%$ \\
Disagree & 292 & $33.07 \%$ \\
Strongly disagree & 70 & $7.93 \%$ \\
\hline
\end{tabular}

Table 11 To what extent do you agree with the following statement: "Webinars should replace face-to-face national and international meetings"

\begin{tabular}{llc}
\hline & $N$ responses & $\%$ responses \\
\hline Strongly agree & 67 & $7.59 \%$ \\
Agree & 164 & $18.57 \%$ \\
Neither agree nor disagree & 222 & $25.14 \%$ \\
Disagree & 330 & $37.37 \%$ \\
Strongly disagree & 100 & $11.33 \%$ \\
\hline
\end{tabular}

The majority of responses were from Surgical Registrars/Residents (51.0\%) and Consultant/Attending surgeons (40.7\%), with a small proportion of Junior Trainees (6.3\%) and Medical Students (2.0\%) (Table 2, Fig. 2).

Over $60 \%$ of respondents stated that prior to COVID19 restrictions they spent on average, zero hours a week attending webinars (Table 3, Fig. 3), whereas following
Table 12 To what extent do you agree with the following statement: "I will continue to attend webinars once COVID-19 restrictions are lifted"

\begin{tabular}{llc}
\hline & $N$ responses & $\%$ responses \\
\hline Strongly agree & 298 & $33.75 \%$ \\
Agree & 500 & $56.63 \%$ \\
Neither agree nor disagree & 64 & $7.25 \%$ \\
Disagree & 15 & $1.70 \%$ \\
Strongly disagree & 6 & $0.68 \%$ \\
\hline
\end{tabular}

Table 13 To what extent do you agree with the following statement: "I would pay to attend webinars given by high-quality speakers"

\begin{tabular}{llc}
\hline & $N$ responses & $\%$ responses \\
\hline Strongly agree & 75 & $8.49 \%$ \\
Agree & 322 & $36.47 \%$ \\
Neither agree nor disagree & 255 & $28.88 \%$ \\
Disagree & 191 & $21.63 \%$ \\
Strongly disagree & 40 & $4.53 \%$ \\
\hline
\end{tabular}

\section{Q1. What is your current level of training?}

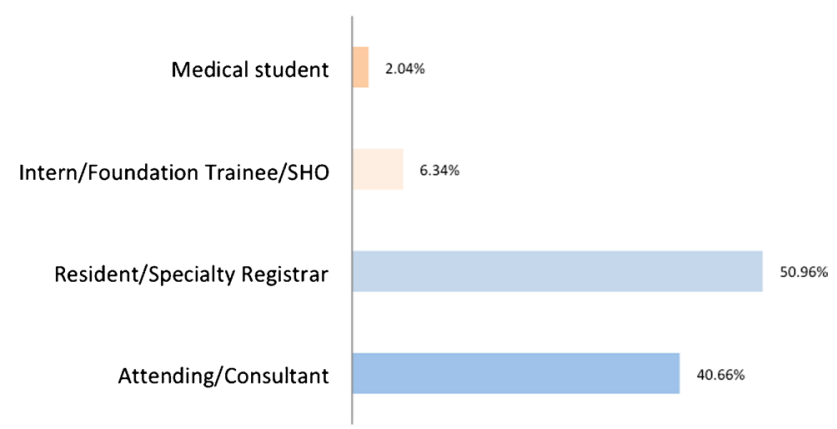

Fig. 2 What is your current level of training?

\section{Q3. Prior to COVID-19 restrictions, how many hours did} you spend attending webinars per week?

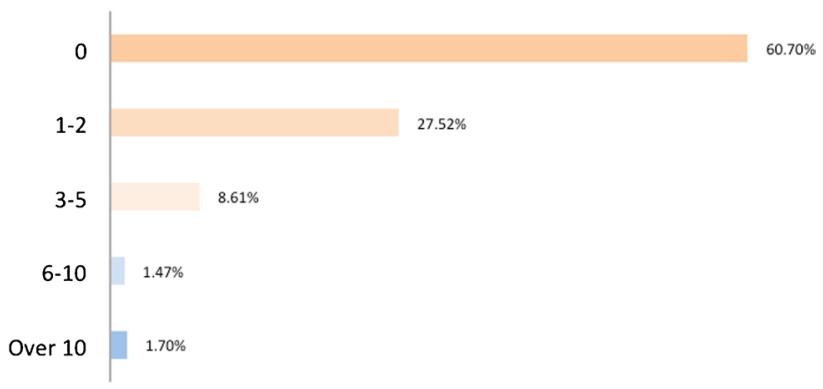

Fig. 3 Prior to COVID-19 restrictions, how many hours did you spend attending webinars per week? 
Q4. Since COVID-19 restrictions, how many hours do you spend attending webinars per week?

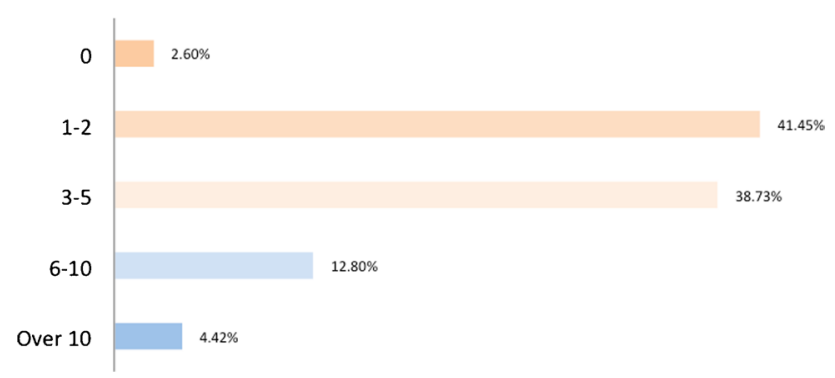

Fig. 4 Since COVID-19 restrictions, how many hours do you spend attending webinars per week?

Q5. Do you mostly attend webinars during the working day or out-of-hours?

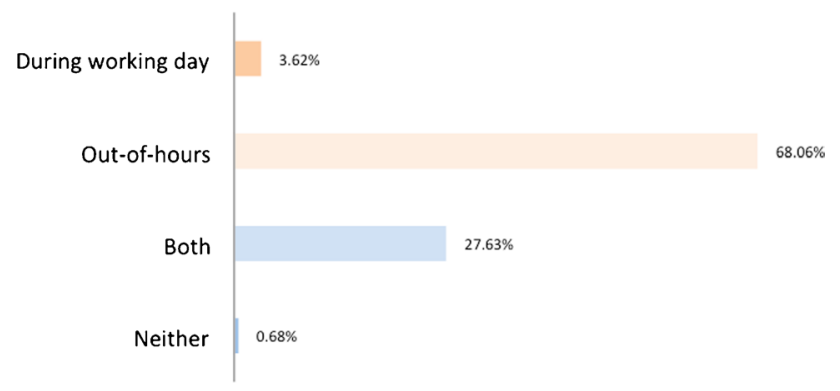

Fig. 5 Do you mostly attend webinars during the working day or outof-hours?

implementation of COVID-19 restrictions, $97.4 \%$ of respondents viewed webinars for at least $1 \mathrm{~h}$ per week (Table 4, Fig. 4). Respondents were more likely to view webinars out of hours (61.8\% solely out-of-hours, $27.6 \%$ both at work and out-of-hours) rather than exclusively during the working day (3.6\%) (Table 5, Fig. 5).

Reasons for attending webinars in order of popularity of answer were as follows: general knowledge/interest, prominent speakers, exam revision, and CPD (Table 6, Fig. 6). Additional reasons identified form the free text responses using thematic analysis principles were as follows: to learn new surgical techniques, keep up to date, and to gain insight into practices in different countries from international speakers.

On the Likert scale (Strongly agree/Agree/Neural/Disagree/Strongly disagree), $77.8 \%$ agreed they learn an equivalent amount from webinars as they do face-to-face lectures (Table 7, Fig. 7). However, $40.5 \%$ voted affirmatively that they prefer live lectures to webinars, with a further $39.5 \%$ selecting the neutral option (Table 8, Fig. 8). Overall, all training grades agreed that they prefer live lectures
Q6. Why do you attend webinars (tick all that apply)

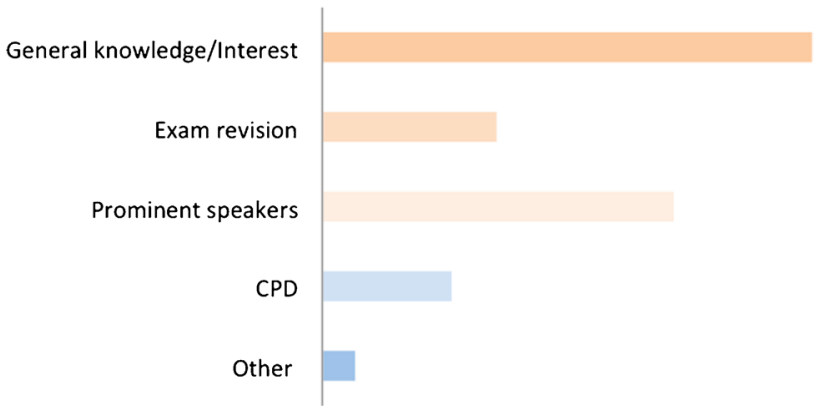

Fig. 6 Why do you attend webinars (tick all that apply)?

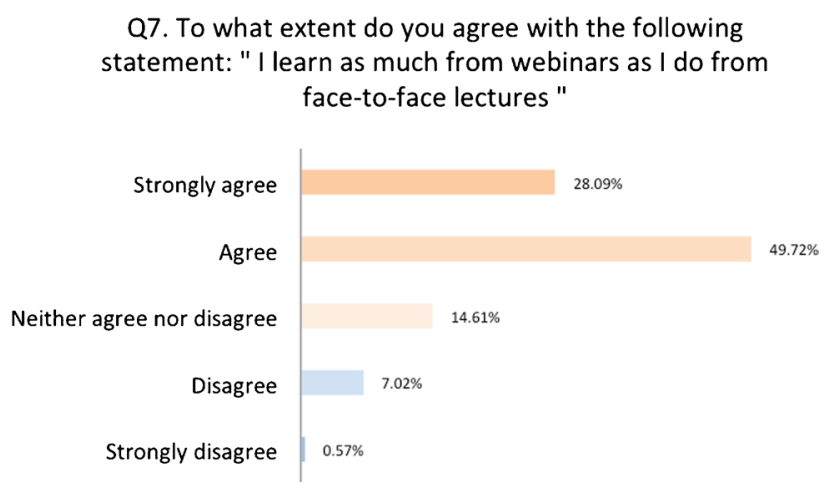

Fig. 7 To what extent do you agree with the following statement: "I learn as much from webinars as I do from face-to-face lectures"

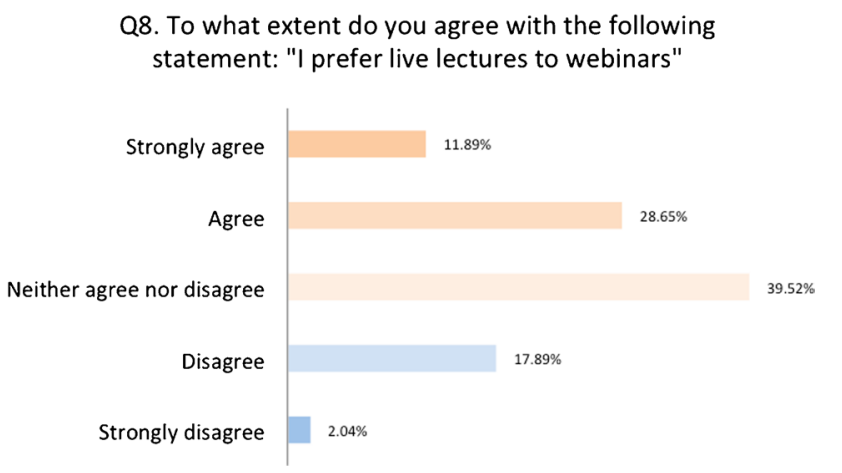

Fig. 8 To what extent do you agree with the following statement: "I prefer live lectures to webinars"

to webinars, with no significant difference between grades (chi ${ }^{2}$ test, $p>0.05$ ).

Over half of respondents reported that they reflect on webinars with colleagues (68.2\%) (Table 9, Fig. 9). Fortyone percent respondents did not believe that webinars should replace face-to-face local teaching, compared to $25.7 \%$ who did. $48.7 \%$ disagreed with the statement that 
Q9. To what extent do you agree with the following statement: "I reflect on/discuss the webinars I attend with colleagues after I watch them"

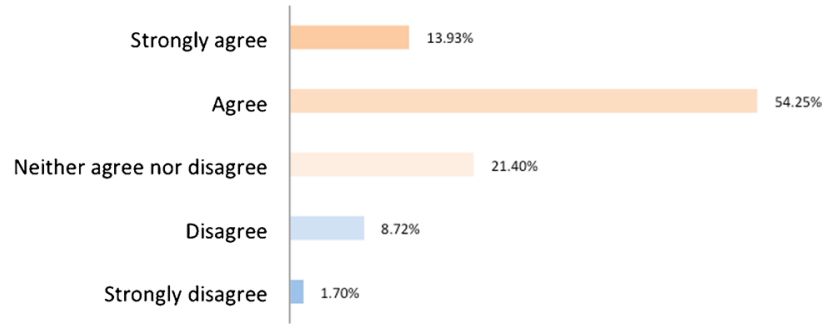

Fig. 9 To what extent do you agree with the following statement: "I reflect on/discuss the webinars I attend with colleagues after I watch them"

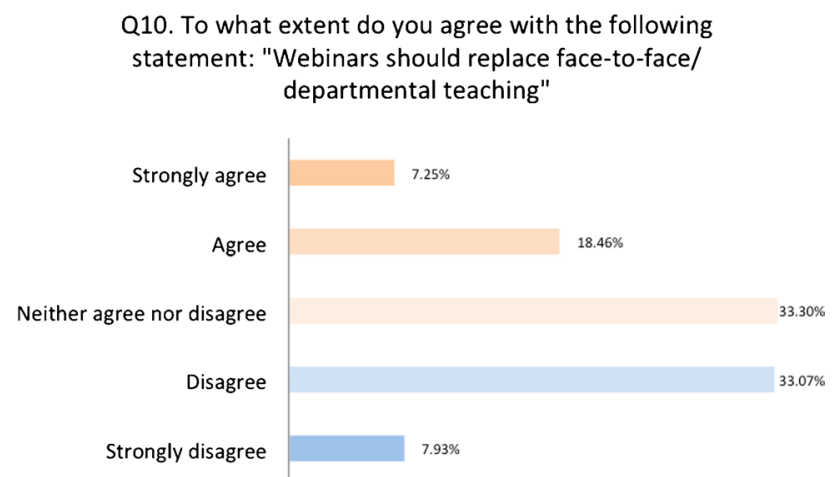

Fig. 10 To what extent do you agree with the following statement: "Webinars should replace face-to-face/departmental teaching"

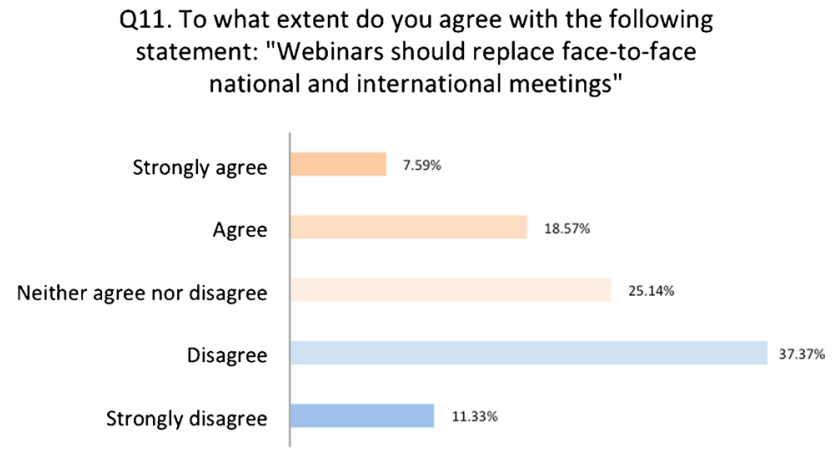

Fig. 11 To what extent do you agree with the following statement: "Webinars should replace face-to-face national and international meetings"

webinars should replace face-to-face international meetings, compared with $26.2 \%$ who agreed (Tables 10 and 11 , Figs. 10 and 11). There was no difference in overall preference for face-to-face learning between training grades (chi ${ }^{2}$ test, $p>0.05$ ).
Q12. To what extent do you agree with the following statement: "I will continue to attend webinars once COVID-19 restrictions are lifted"

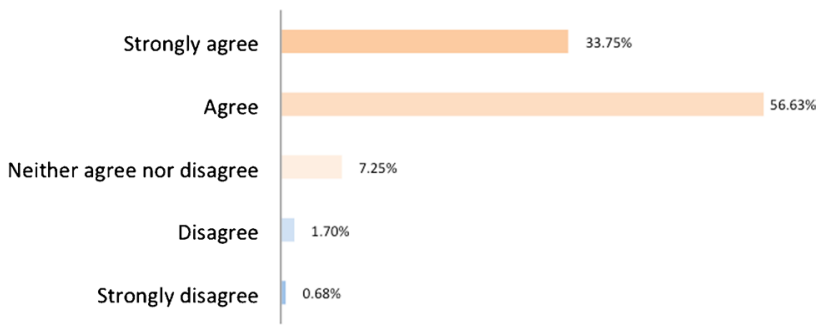

Fig. 12 To what extent do you agree with the following statement: "I will continue to attend webinars once COVID-19 restrictions are lifted"

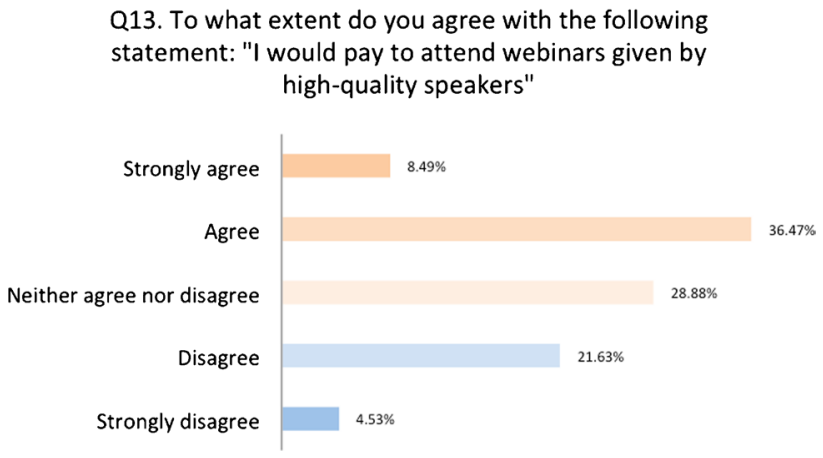

Fig. 13 To what extent do you agree with the following statement: "I would pay to attend webinars given by high-quality speakers"

The vast majority of respondents indicated that they intend to continue utilizing webinars even once COVID-19 restrictions are eased (90.4\% agree versus only $2.4 \%$ disagree) (Table 12, Fig. 12). Additionally $45.0 \%$ indicated that they would pay to attend webinars delivered by high-quality speakers (Table 13, Fig. 13).

\section{Discussion}

Online webinars have played a leading role in filling the void in medical education that has been created by COVID-19 social distancing measures, travel restrictions, and clinical demands on staff. They have enabled surgeons to partake in self-directed learning and stay up to date with international advances, while minimizing the need for spending on travel or teaching materials. [9] The results of this cross-sectional survey indicate that webinar use has significantly increased since the COVID-19 pandemic. Webinars can provide attendees with equivalent learning experience compared to face-to-face sessions, and will continue to be used as an educational resource following future easing of restrictions. 
However, our data also suggests that webinars should ideally be used in conjunction with local teaching and international meetings, rather than acting as a substitute for in-person teaching. Rates of preference for face-to-face versus online learning were similar irrespective of training grade, from medical students to consultants and attending surgeons. Thereby suggesting that despite the success of remote educational models, human interaction continues to be regarded as a fundamental aspect of learning,

Providing evidence of continued professional development (CPD) or continued medical education (CME) is a key component in the appraisal and revalidation of senior surgeons. [10] The introduction of initiatives focusing on safe working hours, such as the European Working Time Directive (EWTD), has capped the number of hours that doctors are permitted to work per week. [11] Compared to other specialties, surgical trainees in particular have felt that the directive has had a negative impact on access to essential training and experience, resulting in a need for self-directed learning outside of working hours. [12] Indeed the results of our survey demonstrate that the majority of respondents access webinars in their own time outside of work. Ease of webinar accessibility makes them a convenient avenue for securing CPD and keeping knowledge up to date. [13] The use of well-known, expert lecturers within webinar series also acts to eliminate concerns that can often arise regarding the validity of virtual resources.

An often-cited advantage of webinars over physical lectures and seminars is that they tend to accumulate minimal costs with regard to organization and delivery. It must be appreciated, however, that there are costs associated with these: the online platform has an annual subscription, for example, Zoom is $£ 192$ per annum at time of writing. Furthermore, there is a time-cost associated with organization of the sessions, hosting them, and, of course, for the speakers who prepare and present their seminars. This should be valued, and offering these at no cost risks devaluing them. However, the overheads are significantly less than face-toface meetings. It could be argued that these savings should be passed on to the attendees.

The financial cost of surgical training is high, with a significant contribution from course and conference fees and travel expenses. [14] The use of more affordable resources for training and professional development may improve inclusion and socioeconomic diversity in surgical trainees. However, the opportunity cost of improved accessibility may be a loss of community and collaboration that is often felt at surgical meetings and conferences. Webinars can provide chances for user interaction and synchronous learning online, but this is difficult to achieve with large virtual audiences. Participants may feel there is a lack of topical discussion that usually promotes depth of learning. $[15,16]$ Nevertheless, the majority of respondents to this survey reported that they reflect on webinars and discuss them with colleagues, which indicates retention of educational content and suggests a degree of transference of the learning to the workplace, despite a largely didactic online method of delivery.

The majority of our sample indicated they feel that they learn as much from webinars as face-to-face learning, although testing this claim was beyond the scope of this study. Despite webinars being subjectively effective, a significant proportion of respondents still would not want webinars to act as a substitute to local or international meetings. This could suggest the "meeting" aspect of meetings holds part of their true value. The documented successful use of webinars in plastic surgery and other surgical specialties has laid down an educational infrastructure that can not only be incorporated into future teaching methods, but also serves as a contingency plan in surgical education in the unfortunate occurrence of similar global circumstances in the future.

While this study does provide a snapshot on surgical opinions on webinar use during COVID-19 and an insight for future applications, it has several limitations. As the sample consisted of those who had registered for the webinar series, it may overestimate engagement with online webinars amongst plastic surgery doctors in general. In addition, as the viewership consisted largely of specialty trainees and senior surgeons, the data does not provide much indication of the usefulness of webinars to students and more junior doctors. It may be that in the earlier stages of surgical training, more interactive environments are conducive to learning. Furthermore, although an international audience completed the survey, the majority of responses from a single country were from the UK. Therefore, there may be cultural factors influencing the readiness to incorporate web-based learning in the future including access to smartphones and reliable broadband.

\section{Conclusions}

Webinars are regarded as effective and sustainable platforms for education by plastic surgeons and surgical trainees. As the world continues to adapt to life after the COVID-19 pandemic, amalgamation of webinars into surgical curriculums can help to capture a larger audience and can compliment traditional teaching methods. Future educational models are likely to be a blended hybrid of physical and remote learning.

Author contribution All authors contributed to the study conception and design. Siri Gowda and Vimal Gokani performed material preparation, data collection, and analysis. Siri Gowda wrote the first draft of the manuscript and all authors commented on previous versions of the manuscript. All authors read and approved the final manuscript. 
Data availability All survey data available on request.

Code availability Not applicable.

\section{Declarations}

Ethics approval All procedures performed in studies involving human participants were in accordance with the ethical standards of the institutional and/or national research committee and with the 1964 Helsinki Declaration and its later amendments or comparable ethical standards. This is a survey-based study. The Local Research Ethics Committee has confirmed that no ethical approval is required.

Consent to participate All survey participants consented to participation.

Consent for publication All survey participants consented to publication of anonymized results.

Conflict of interest Siri Gowda, Jonathan Simmons, and Vimal J Gokani declare no competing interests.

\section{References}

1. Hari Prasetyono TO, Christian A (2020) Multiscreen to screen webinar for education beyond border: a review. Ann Med Surg 59. https://doi.org/10.1016/j.amsu.2020.09.041

2. Payne KFB, Wharrad H, Watts K (2012) Smartphone and medical related App use among medical students and junior doctors in the United Kingdom (UK): a regional survey. BMC Med Inform Decis Mak 12(1). https://doi.org/10.1186/1472-6947-12-121

3. Al-Hadithy N, Ghosh S (2013) Smartphones and the plastic surgeon. J Plast Reconstr Aesthetic Surg 66(6). https://doi.org/10. 1016/j.bjps.2013.02.014

4. Waltzman JT, Tadisina KK, Zins JE (2016) The rise of technology in plastic surgery education: is the textbook dead on arrival (DOA)? Aesthetic Surg J 36(2). https://doi.org/10.1093/asj/sjv144

5. Fadlelmola FM, Panji S, Ahmed AE et al (2019) Ten simple rules for organizing a webinar series. PLoS Comput Biol 15(4). https:// doi.org/10.1371/journal.pcbi.1006671

6. Martin-Smith JD, McArdle A, Carroll SM, Kelly EJ (2015) Webinar: a useful tool in plastic surgery specialty trainee education.
J Plast Reconstr Aesthetic Surg 68(9). https://doi.org/10.1016/j. bjps.2015.05.034

7. Reissis D, Joji N, Campbell E, Sharma VP, Staruch RMT, Baker BG. PLASTA National Webinar Series: a developing model for remote surgical education. J Plast Reconstr Aesthetic Surg 73(8). https://doi.org/10.1016/j.bjps.2020.05.008

8. Patel NM, Khajuria A, Khajuria A (2020) Utility of a webinar to educate trainees on UK core surgical training (CST) selection - a cross sectional study and future implications amidst the COVID19 pandemic. Ann Med Surg 59. https://doi.org/10.1016/j.amsu. 2020.08 .054

9. Mukhtar K, Javed K, Arooj M, Sethi A (2020) Advantages, limitations and recommendations for online learning during covid-19 pandemic era. Pakistan J Med Sci 36(COVID19-S4). https://doi. org/10.12669/pjms.36.COVID19-S4.2785

10. Stewart GD, Teoh KH, Pitts D, Garden OJ, Rowley DI (2008) Continuing professional development for surgeons. Surgeon 6(5). https://doi.org/10.1016/S1479-666X(08)80053-4

11. Maybury C (2014) The European Working Time Directive: a decade on. Lancet 384(9954). https://doi.org/10.1016/S01406736(14)61972-3

12. Lambert TW, Smith F, Goldacre MJ (2016) The impact of the European Working Time Directive 10 years on: views of the UK medical graduates of 2002 surveyed in 2013-2014. JRSM Open 7(3). https://doi.org/10.1177/2054270416632703

13. Knipfer C, Wagner F, Knipfer K, Millesi G, Acero J, Hueto JA, Nkenke E (2019) Learners' acceptance of a webinar for continuing medical education. Int J Oral Maxillofac Surg 48(6):841-846. https://doi.org/10.1016/j.ijom.2018.11.010

14. O'Callaghan J, Mohan HM, Sharrock A et al (2017) Cross-sectional study of the financial cost of training to the surgical trainee in the UK and Ireland. BMJ Open 7(11). https://doi.org/10.1136/ bmjopen-2017-018086

15. Ebner C, Gegenfurtner A (2019) Learning and satisfaction in webinar, online, and face-to-face instruction: a meta-analysis. Front Educ 4. https://doi.org/10.3389/feduc.2019.00092

16. Gegenfurtner A, Zitt A, Ebner C (2020) Evaluating webinar-based training: a mixed methods study of trainee reactions toward digital web conferencing. Int J Train Dev 24(1). https://doi.org/10.1111/ ijtd.12167

Publisher's note Springer Nature remains neutral with regard to jurisdictional claims in published maps and institutional affiliations. 